\title{
Editorial for special issue on epidemiology and prevention of prostate cancer
}

\author{
Ian M. Thompson • Donna P. Ankerst
}

Received: 10 January 2012/ Accepted: 13 January 2012/Published online: 22 January 2012

(C) Springer-Verlag 2012

We are very proud of the quality and range of the contributions from the authors of this edition of World Journal of Urology with a specific focus on the epidemiology and prevention of prostate cancer. This disease is a conundrum for governments and healthcare agencies across the globe: while they all aspire to increase life expectancy from any number of public health measures, as longevity increases, so does the risk of prostate cancer diagnosis and mortality. The organized response to this expanding risk of disease has frequently been an increased focus on early detection, generally with the monolithic use of prostate-specific antigen (PSA) and digital rectal examination (DRE), using $>4 \mathrm{ng} / \mathrm{mL}$ and 'abnormal' as indications for biopsy and intervention with a goal to reduce mortality. While in some regions and countries, within years of the inception of this approach, a fall in prostate cancer mortality has been seen, a concurrent enormous increase in treatment has also been the result with substantial reallocation of resources as well as the growth of huge populations of prostate cancer survivors, almost all of whom have at least some change in their quality of life to show for their experience.

The fundamental two challenges with prostate cancer are its prevalence and its range of behaviors. Regarding the former, the prevalence of the disease in an individual man is very close to his age. For example, about $60 \%$ of 60 -year-olds harbor what would be deemed 'prostate cancer cells' in their prostate if fully examined histologically. As the lifetime risk of prostate cancer mortality ranges

I. M. Thompson · D. P. Ankerst $(\bowtie)$

Department of Urology, UTHSCSA, 7703 Floyd Curl Dr.,

San Antonio, TX 78229, USA

e-mail: ankerst@uthscsa.edu

I. M. Thompson

e-mail: thompsoni@uthscsa.edu from 3 to $4 \%$, it is clear that the bulk of these prostate cancers are not truly 'cancer' phenotypically, but only histologically. Similarly, some prostate cancers are quite aggressive, developing into systemic disease early on; fortunately, this is uncommon. Again, the challenge for well-intentioned clinicians and healthcare agencies is that, in the search for the tumors that will take a man's life, we inadvertently (and more commonly) stumble on tumors that will not. Historically, in the United States, prostate cancer has often been viewed monolithically and treated as if all of these 'tumors' behaved as 'cancers'. Thus, over $90 \%$ of low-risk cancers received radical surgery or radiation [1].

To the reader of this issue, it is clear that this approach is inappropriate, especially given the level of knowledge we have today. A far more rational approach would be as follows:

1. Identify the man who is at a very low risk of prostate cancer over a decade or more. Instruct him to 'be checked' a decade from now.

2. For the man who is at a higher risk of prostate cancer, consider an intervention to prevent the development of the disease. Ideally, the preventive strategy should target pathways of carcinogenesis in an individual man.

3. In the man for whom such a prevention strategy is ongoing, recognizing that such prevention efforts will reduce the risk of disease but not eliminate it, monitor his risk for cancer with a range of biomeasures and biomarkers.

4. If, during the monitoring process, testing indicates the potential presence of an indolent (low risk) tumor, no detection procedures (i.e., biopsy) are performed. Conversely, if testing indicates the potential presence 
of an aggressive, high-risk tumor, biopsy is performed. If an aggressive tumor is identified, initiate the optimal therapy for this tumor.

While these ideas might seem a thing of the future, they are possible today for all of these steps.

1. Our colleague authors show that if PSA is sufficiently low at a young age, men probably do not need testing for many years. This step then 'unburdens' PSA testing in this very low-risk population. It also significantly reduces the risk of a spurious elevation in PSA leading to a biopsy recommendation.

2. Prevention strategies are now proven, specifically with five alpha-reductase inhibitors. There are risks and benefits, and not all men will opt to pursue these. Other strategies like selenium and vitamin $\mathrm{E}$ are now clearly ineffective and may indeed cause harm. Diet and physical activity are very promising opportunities and obviously have the added value of reducing the risk of multiple diseases concurrently.

3. We now have several risk assessment tools that are very effective in assessing a man's risk of cancer. Importantly, these tools incorporate not just a range of current and innovative biomarkers from serum and urine but also 'biomeasures' that are very important predictors of risk. In our clinics at the UT Health Science Center at San Antonio and in many comparable institutions around the world, it is now standard that at-risk men routinely are assessed with a risk assessment tool such as the Prostate Cancer Prevention Trial Risk Calculator (www.prostate.cancer.risk. calculator.com). Even for those of us who were involved in developing these risk assessment tools, we find that the accuracy of the risk helps us communicate far more effectively to the patient.

4. Most importantly, both our risk assessment tools as well as our new biomarkers are increasingly important in assessing a man's risk of aggressive (high grade) cancer as compared to his risk of potentially indolent disease. For example, a man with a $25 \%$ risk of prostate cancer but a $4 \%$ risk of high-grade disease can be told that there is a 1-in-4 risk of cancer but that the risk of cancer that is most likely dangerous to him is only 1 in 25 . Similarly, in this case, he is then told that if a cancer is found, there is a 5.5-fold greater likelihood that it is a cancer that may be inconsequential. If such a tumor is found, some might look upon it as a 'good thing to know'. Many patients who, after examining treatment options and decide on active surveillance, then reflect on the burden of surveillance and ask themselves 'might I have been better off by not knowing in the first place?' On the other hand, a man such as one of us (IMT) saw in clinic today whose risk of cancer is $60 \%$ and risk of high-grade disease is $20 \%$ would have a completely different discussion; out of five men biopsied, two will not have cancer, about two will have lower risk cancer and one will have aggressive cancer. Thus, there is a 1-in-3 chance that a cancer found will be important and may require treatment.

It would be a fascinating exercise to assemble this group of experts from this issue in a room and charge them with designing a 2012-era randomized trial of screening for prostate cancer. It is our suspicion that, with our knowledge today, we could indeed witness a reduction in prostate cancer mortality with far fewer individuals screened and treated. We suspect that all of us wish we knew then (about 1990) what we know now vis-a-vis biomarker science when both the PLCO and ERSPC screening trials were being designed.... For now, it is all of our responsibilities to practice state-of-the-art prostate cancer control. It is through the scientific efforts of these authors and many others that we should see continued falls in prostate cancer mortality while concurrently reducing the burden of screening and treatment.

\section{Reference}

1. Cooperberg MR, Broering JM, Carroll PR (2010) Time trends and local variation in primary treatment of localized prostate cancer. J Clin Oncol 28:1117-1123 